Louisiana State University

LSU Digital Commons

Faculty Publications

Department of Physics \& Astronomy

$12-1-2004$

\title{
The massive neutron star or low-mass black hole in 2S 0921-630
}

T. Shahbaz

Instituto Astrofisico de Canarias

J. Casares

Instituto Astrofisico de Canarias

C. A. Watson

The University of Sheffield

P. A. Charles

University of Southampton

R. I. Hynes

The University of Texas at Austin

See next page for additional authors

Follow this and additional works at: https://digitalcommons.Isu.edu/physics_astronomy_pubs

\section{Recommended Citation}

Shahbaz, T., Casares, J., Watson, C., Charles, P., Hynes, R., Shih, S., \& Steeghs, D. (2004). The massive neutron star or low-mass black hole in 2S 0921-630. Astrophysical Journal, 616 (2 II) https://doi.org/ $10.1086 / 426504$

This Article is brought to you for free and open access by the Department of Physics \& Astronomy at LSU Digital Commons. It has been accepted for inclusion in Faculty Publications by an authorized administrator of LSU Digital Commons. For more information, please contact ir@lsu.edu. 


\section{Authors}

T. Shahbaz, J. Casares, C. A. Watson, P. A. Charles, R. I. Hynes, S. C. Shih, and D. Steeghs 


\section{递/ QUEEN'S \\ - UNIVERSITY BELFAST

\section{The massive neutron star or low-mass black hole in 2S 0921-630}

Shahbaz, T., Casares, J., Watson, C., Charles, P. A., Hynes, R. I., Shih, S. C., \& Steeghs, D. (2004). The massive neutron star or low-mass black hole in 2S 0921-630. Astrophysical Journal, 616(2 II), L123-L126.

Published in:

Astrophysical Journal

Queen's University Belfast - Research Portal:

Link to publication record in Queen's University Belfast Research Portal

\section{General rights}

Copyright for the publications made accessible via the Queen's University Belfast Research Portal is retained by the author(s) and / or other copyright owners and it is a condition of accessing these publications that users recognise and abide by the legal requirements associated with these rights.

Take down policy

The Research Portal is Queen's institutional repository that provides access to Queen's research output. Every effort has been made to ensure that content in the Research Portal does not infringe any person's rights, or applicable UK laws. If you discover content in the Research Portal that you believe breaches copyright or violates any law, please contact openaccess@qub.ac.uk. 
The Astrophysical Journal, 616:L123-L126, 2004 December 1

(C) 2004. The American Astronomical Society. All rights reserved. Printed in U.S.A.

THE MASSIVE NEUTRON STAR OR LOW-MASS BLACK HOLE IN 2S 0921-630

\author{
T. Shahbaz, ${ }^{1}$ J. Casares, ${ }^{1}$ C. A. Watson, ${ }^{2}$ P. A. Charles, ${ }^{3}$ R. I. Hynes, ${ }^{4}$ S. C. Shih, ${ }^{3}$ and D. Steeghs ${ }^{5}$ \\ Received 2004 August 6; accepted 2004 October 6; published 2004 October 25
}

\begin{abstract}
We report on the optical spectroscopy of the eclipsing halo low-mass X-ray binary 2S 0921-630, which reveals the absorption-line radial velocity curve of the K0 III secondary star with a semiamplitude $K_{2}=92.89 \pm 3.84 \mathrm{~km}$ $\mathrm{s}^{-1}$, a systemic velocity $\gamma=34.9 \pm 3.3 \mathrm{~km} \mathrm{~s}^{-1}$, and an orbital period $P_{\text {orb }}$ of $9.0035 \pm 0.0029$ days ( $1 \sigma$ ). Given the quality of the data, we find no evidence for the effects of X-ray irradiation. Using the previously determined rotational broadening of the mass donor and applying conservative limits on the orbital inclination, we constrain the compact object mass to be $2.0-4.3 M_{\odot}(1 \sigma)$, ruling out a canonical neutron star at the $99 \%$ level. Since the nature of the compact object is unclear, this mass range implies that the compact object is either a low-mass black hole with a mass slightly higher than the maximum possible neutron star mass $\left(2.9 M_{\odot}\right)$ or a massive neutron star. If the compact object is a black hole, it confirms the prediction of the existence of low-mass black holes, while if the object is a massive neutron star, its high mass severely constrains the equation of state of nuclear matter.
\end{abstract}

Subject headings: black hole physics — stars: individual (V395 Carinae) — stars: neutron $\mathrm{X}$-rays: binaries — X-rays: individual (2S 0921-630)

\section{INTRODUCTION}

A knowledge of the neutron star mass distribution provides a fundamental test of theories of the equation of state of nuclear matter, the applicability of general relativity as the correct theory of gravity, and information on the evolutionary history of the progenitor stars. To date, only studies of radio pulsars have provided accurate mass determinations of neutron stars, reflecting their mass at formation, $1.35 \pm 0.04 M_{\odot}$ (van Kerkwijk 2001). Although masses can also be obtained from accreting X-ray pulsars, there are large uncertainties. In high-mass X-ray binaries (HMXBs) there are uncertainties due to non-Keplerian perturbations in the radial velocity curves caused by effects such as stellar wind contamination and X-ray heating. In low-mass Xray binaries (LMXBs), the situation is worse because the intense $\mathrm{X}$-ray irradiation usually suppresses the light from the donor (Charles \& Coe 2004). It is only in a few exceptional cases in which the companion is evolved (and hence more luminous), or during X-ray off-states, that dynamical information can be extracted about the nature of the compact object.

The SAS-3 X-ray source, 2S 0921-630, was identified with a $\sim 16$ mag blue star (Li et al. 1978), V395 Car, whose optical spectrum was characteristic of LMXBs (Branduardi-Raymont et al. 1983). EXOSAT observations showed a broad ( $>1$ day), shallow $\mathrm{X}$-ray eclipse, during which the spectrum softened, allowing an estimate of the size of the accretion disk corona (ADC; Mason et al. 1987). Only a handful of ADC sources are known in which the compact object is permanently obscured from our line of sight by the accretion disk. Limited optical photometry and emissionline spectroscopy (Cowley et al. 1982; Branduardi-Raymont et al. 1983) shows that $2 \mathrm{~S} 0921-630$ has an orbital period of 9.02 days and $(\sim 1 \mathrm{mag})$ dips, where the $(B-V)$ reddening by up to

\footnotetext{
${ }^{1}$ Instituto de Astrofísica de Canarias, 38200 La Laguna, Tenerife, Spain; tsh@11.iac.es, jcv@11.iac.es.

${ }^{2}$ Department of Physics and Astronomy, University of Sheffield, Sheffield S3 7RH, England; c.watson@sheffield.ac.uk.

${ }^{3}$ School of Physics and Astronomy, University of Southampton, Southampton SO17 1BJ, UK; pac@soton.ac.uk, icshih@soton.ac.uk.

${ }^{4}$ McDonald Observatory and Department of Astronomy, University of Texas at Austin, 1 University Station C1400, Austin, TX 78712; rih@astro.as.utexas.edu.

${ }^{5}$ Harvard-Smithsonian Center for Astrophysics, 60 Garden Street, MS-67, Cambridge, MA 02138; steeghs@cfa.harvard.edu.
}

0.4 mag has been interpreted as the eclipse of the disk by the latetype star (Chevalier \& Ilovaisky 1982). High-resolution optical spectroscopy revealed the donor to be a K0 III star with a rotational velocity of $v \sin i=65 \pm 9 \mathrm{~km} \mathrm{~s}^{-1}$, contributing $\sim 25 \%$ to the observed flux (Shahbaz et al. 1999, hereafter S99). 2S 0921-630 is one of those rare LMXBs in which the secondary is visible despite the presence of a luminous disk.

Since there has been no detection of type I X-ray bursts of pulsations, the nature of the compact object is unclear. In this Letter we present the results of an intensive campaign to measure the dynamical mass of the compact object in 2S 0921-630.

\section{OBSERVATIONS AND DATA REDUCTION}

Time-resolved spectroscopic observations of 2S 0921-630 were obtained on the $1.9 \mathrm{~m}$ telescope at the South African Astronomical Observatory (SAAO) during 2003 March 30 to April 18. Grating 5 (1200 lines $\mathrm{mm}^{-1}$ ) was used, centered at $4900 \AA$ and covering a wavelength range of 4529-5377 $\AA$ with a dispersion of $0.49 \AA$ pixel $^{-1}$. A 2".0 slit gave a spectral resolution of $1.1 \AA\left(=64 \mathrm{~km} \mathrm{~s}^{-1}\right.$ at $\left.5200 \AA\right)$ as measured from the $\mathrm{Cu}$-Arc arc lines. In general, the conditions were good, with the seeing varying between $1^{\prime \prime}$ and $3^{\prime \prime}$. A total of 83 spectra were obtained with an exposure time of $1800 \mathrm{~s}$. Template field stars of a variety of spectral types were also observed. The images were debiased and flat-fielded, and the one-dimensional spectra were extracted using the standard method.

In addition, 34 spectra were also obtained at the Very Large Telescope (VLT), New Technology Telescope (NTT), AngloAustralian Telescope (AAT; Australia), and Magellan Telescope as part of a long-term project on X-ray binaries. A total of nine FORS2 spectra with exposure times in the range 300-900 s were obtained on the nights of 2003 May 18 and June 22-23. The $\mathrm{R} 1400 \mathrm{~V}$ grating was used in combination with a 0 ".7 slit resulting in a wavelength coverage of 4514-5815 $\AA$ and a resolution of $70 \mathrm{~km} \mathrm{~s}^{-1}$. Instrumental flexure was monitored by cross-correlating the sky spectra and was found to be very small, always within $6 \mathrm{~km} \mathrm{~s}^{-1}$. Eleven Magellan spectra were obtained during 2003 December 12-16 with the Baade telescope at Las Campanas using the Inamori Magellan Areal Camera and Spectrograph in the longcamera mode using an exposure time of $300 \mathrm{~s}$. The 600 grating together with a 0.7 long slit provided spectra covering 3720- 


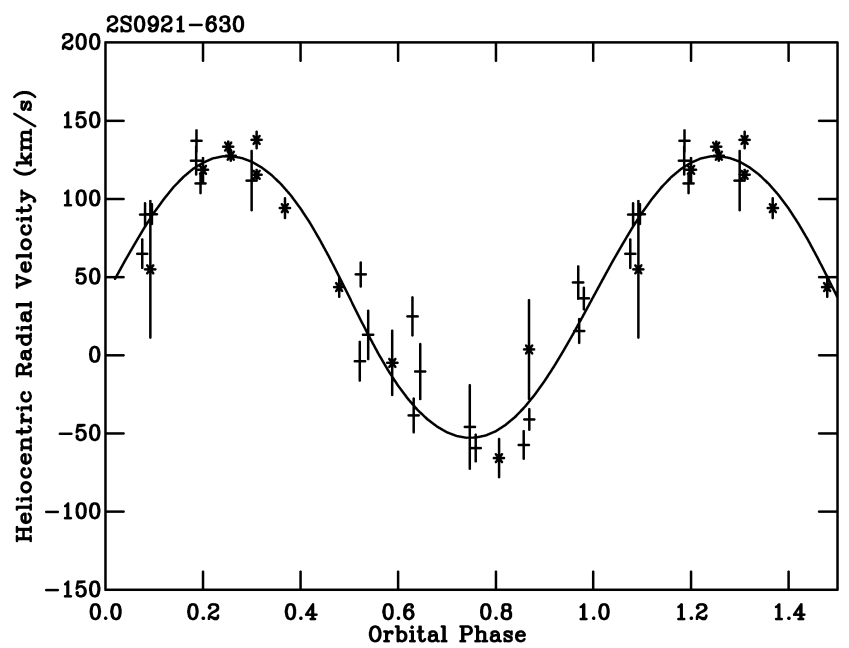

FIG. 1.-Radial velocity curve of the secondary star. The plus signs show the SAAO data, and the asterisks show the VLT, NTT, AAT, and Magellan data. The solid line shows a sinusoidal fit. The data have been phase-folded, and 1.5 orbital cycles are shown for clarity.

$6830 \AA$ with a dispersion of $0.75 \AA$ pixel $^{-1}$. Ten AAT spectra were obtained using the Royal Greenwich Observatory spectrograph on the nights of 2002 June 6-11 with an exposure time of $1800 \mathrm{~s}$. The R1200B grating centered at $4350 \AA$ together with a 1 ".0 slit provided spectra covering $3500-5250 \AA \AA$ with a resolution of $70 \mathrm{~km} \mathrm{~s}^{-1}$. A total of four NTT spectra were obtained with the ESO Multimode Instrument and grating 6 on the nights of 2002 June 8-10 with exposure times of 600-900 s. The spectra cover the spectral range $4400-5150 \AA$ with a resolution of $75 \mathrm{~km} \mathrm{~s}^{-1}$. The AAT and NTT data reduction details are given in Casares et al. (2003).

\section{THE RADIAL VELOCITY CURVE AND SPECTRUM}

To increase the signal-to-noise ratio of the spectra prior to crosscorrelation, the individual spectra were variance-averaged into nightly means or groups of $\sim 3 \mathrm{hr}$, depending on the quality of the individual spectra. The spectra were interpolated onto a constant velocity scale $\left(32 \mathrm{~km} \mathrm{~s}^{-1}\right.$ pixel $\left.^{-1}\right)$ and normalized by fitting a spline function to the continuum. A total of 31 absorption-line radial velocities were measured by cross-correlation with a template star. The region $5100-5300 \AA$ common to all the spectra, primarily containing the $\mathrm{Mg}$ I $(5167.3,5172.7$, and $5183.6 \AA$ ) absorption blend, was used in the analysis. The template star's radial velocity, determined using the position of the $\mathrm{Fe}$ I $\lambda 4957.597$ absorption line, was then added to the radial velocities of $2 \mathrm{~S}$ 0921-630. The resulting radial velocity data (see Fig. 1) were then fitted with a sinusoidal function. Using spectral type template stars in the range G6-K4 III, we obtain $K_{2}$-values in the range 92-99 $\mathrm{km} \mathrm{s}^{-1}$ (see Table 1). The secondary star's spectral type

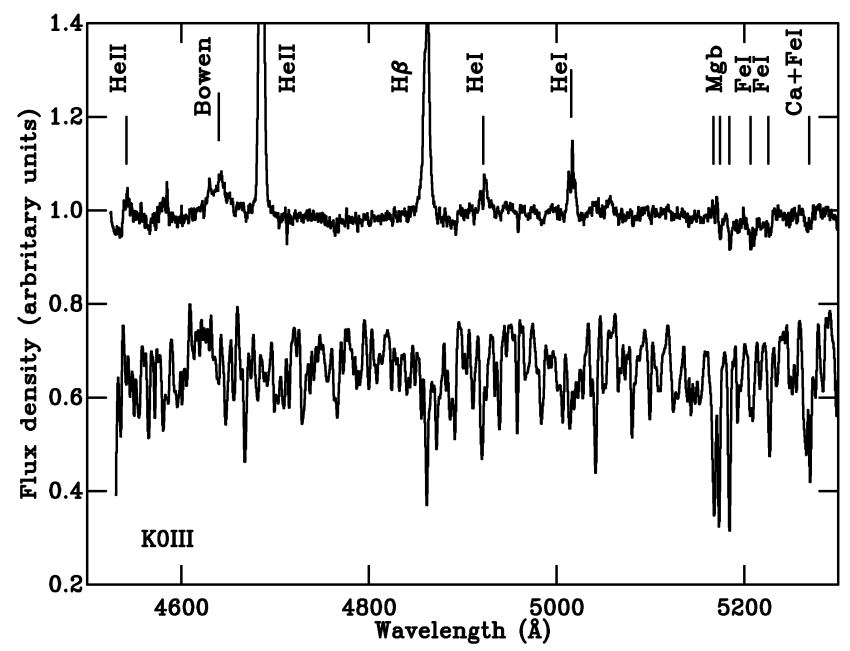

FIG. 2.-Top: Variance-weighted Doppler-shifted average spectrum of 2S 0921-630 in the rest frame of the secondary star. The $\mathrm{H}$ and $\mathrm{He}$ emission lines arising from the accretion disk are clearly seen. Bottom: K0 III template star HD 82565 . The spectra have been normalized and shifted vertically for clarity.

has previously been determined from high-resolution spectroscopy to be K0 III (S99), and our work confirms this. Fitting the radial velocities with a K0 III template star, we find a minimum reduced $\chi^{2}$ of 4.3 with the best-fit parameters $\gamma=34.9 \pm 3.3 \mathrm{~km} \mathrm{~s}^{-1}$, $K_{2}=92.89 \pm 3.84 \mathrm{~km} \mathrm{~s}^{-1}, P_{\text {orb }}=9.0035 \pm 0.0029$ days, and $T_{0}=$ HJD 2,453,099.51 \pm 0.08 , where $P_{\text {orb }}$ is the orbital period, $T_{0}$ is the time at phase 0.0 defined as the inferior conjunction of the secondary star, $\gamma$ is the systemic velocity, and $K_{2}$ is the radial velocity semiamplitude ( $1 \sigma$ errors are quoted with the error bars rescaled so that the reduced $\chi^{2}$ of the fit is 1 ). Our ephemeris is consistent with that determined by Mason et al. (1987). We also computed a $\chi^{2}$ periodogram of the data to investigate the significance of other periods. No significant peaks (at $>99.99 \%$ level) were present other than the one at 9.0035 days. Finally, to check if the 9.0035 day period is affected by an alias, we computed the window function; no significant peaks were present at 9.0035 days. Fitting the radial velocity data with an eccentric orbit did not yield a better fit; the eccentric fit was significant only at the $38 \%$ level.

At a distance of $\sim 10 \mathrm{kpc}$ (Cowley et al. 1982), 2S 0921-630 lies $1.6 \mathrm{kpc}$ below the Galactic plane and so belongs to the halo population. Note that the systemic velocity is not consistent with the radial velocity because of Galactic differential rotation, $\sim 60 \mathrm{~km} \mathrm{~s}^{-1}$ at $\sim 10 \mathrm{kpc}$ (Dehnen \& Binney 1998). However, what is interesting is that the magnitude of the systemic velocity seems to be about a factor of $\sim 4$ lower compared to other neutron star halo systems (Casares et al. 1998, 2002; Torres et al. 2002).

In Figure 2 we show the variance-weighted Doppleraveraged spectrum. The strongest features are the $\mathrm{H}, \mathrm{He}$ emission

TABLE 1

Radial Velocity Curve fits ( $1 \sigma$; HJD 2,453,099+)

\begin{tabular}{|c|c|c|c|c|c|c|c|}
\hline Template & Spectral Type & $\begin{array}{c}\gamma \\
\left(\mathrm{km} \mathrm{s}^{-1}\right)\end{array}$ & $\begin{array}{c}P_{\text {orb }} \\
\text { (day) }\end{array}$ & $T_{0}^{*}$ & $\begin{array}{c}K_{2} \\
\left(\mathrm{~km} \mathrm{~s}^{-1}\right)\end{array}$ & $\chi_{\nu}^{2}$ & $\underset{(\%)}{f}$ \\
\hline HD 122571 & G6 III & $33.3(3.8)$ & $9.0019(36)$ & $9.54(10)$ & 95.1(4.4) & 5.8 & 6.7 \\
\hline HD 63513. & G7 III & $34.2(3.6)$ & $9.0017(32)$ & $9.53(9)$ & $94.2(3.7)$ & 5.3 & 7.8 \\
\hline HD 40359 & G8 III & $36.9(3.1)$ & $9.0040(30)$ & $9.51(9)$ & $92.2(3.7)$ & 4.5 & 8.6 \\
\hline HD 71863 & G9 III & $35.1(3.3)$ & $9.0027(29)$ & $9.51(8)$ & $93.9(3.8)$ & 4.3 & 9.6 \\
\hline HD 82565 & K0 III & $34.9(3.3)$ & $9.0035(29)$ & $9.51(8)$ & $92.9(3.8)$ & 4.3 & 9.9 \\
\hline HD 39523 & K1 III & $37.6(3.2)$ & $9.0036(28)$ & $9.53(7)$ & $94.3(3.7)$ & 4.6 & 9.0 \\
\hline HD 61248 & K3 III & $41.0(3.5)$ & $9.0062(32)$ & $9.58(7)$ & $96.5(4.1)$ & 6.1 & 7.9 \\
\hline HD $40522 \ldots \ldots$ & K4 III & $42.0(3.7)$ & $9.0057(34)$ & $9.59(7)$ & $98.6(4.3)$ & 6.0 & 8.2 \\
\hline
\end{tabular}




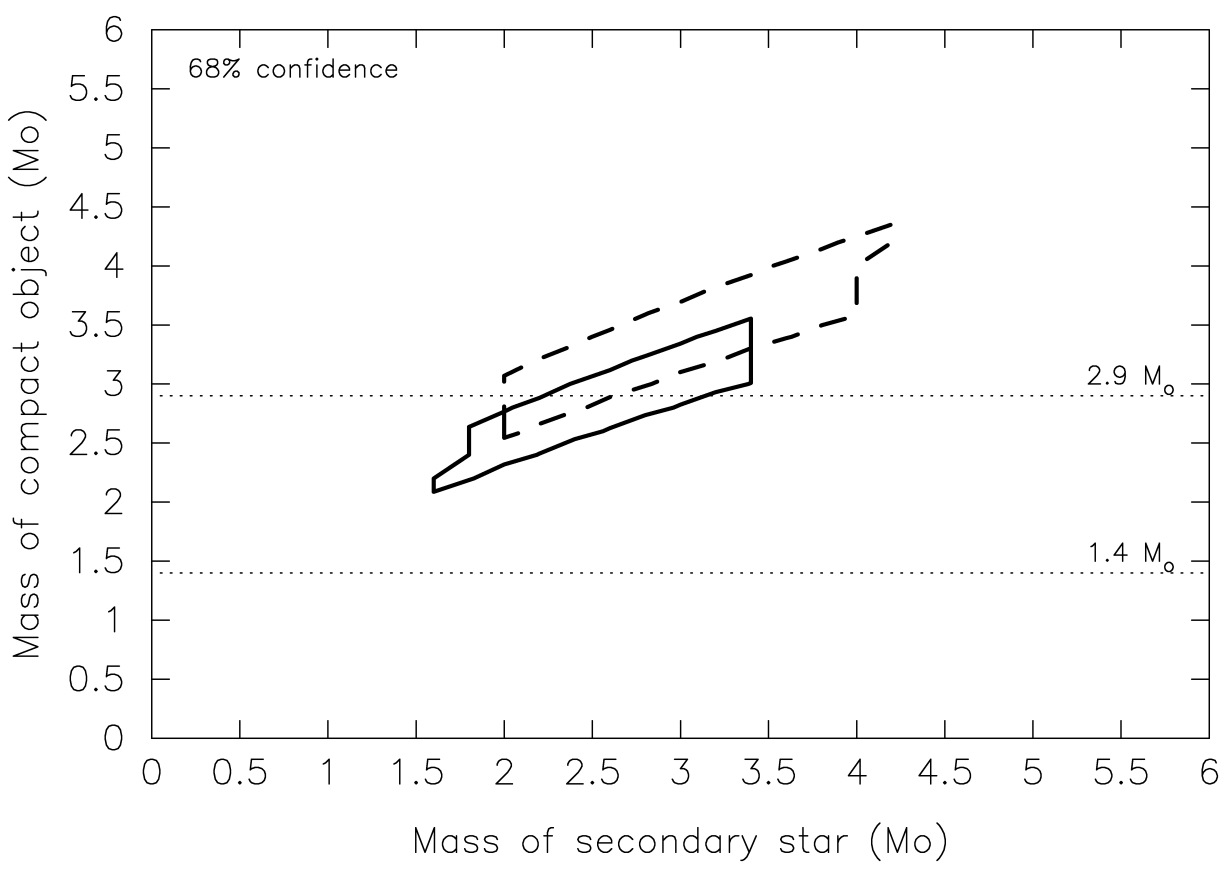

FIG. 3.-Irradiation model $L_{\mathrm{X}}=2 \times 10^{35} \mathrm{ergs} \mathrm{s}^{-1}$. The effect of X-ray heating on the secondary star's radial velocity curve. The solid and dashed contours show the $68 \%$ confidence level for $i=90^{\circ}$ and $70^{\circ}$, respectively. The dotted lines mark the canonical and maximum neutron star mass.

lines, which appear single peaked, even at the resolution of these data. Weak absorption features of $\mathrm{Mg}$ I $\lambda 5175$ and the $\mathrm{Ca} / \mathrm{Fe}$ blend at $5269 \AA$ allow us to determine the fraction of light from the secondary star. We find that the secondary star contributes $\sim 10 \%$ to the observed flux at $5200 \AA$ (see Table 1).

\section{THE MASS OF THE COMPACT OBJECT}

The observed X-rays are reflected into our line of sight by scattering. Although the observed $\mathrm{X}$-ray luminosity is low, $L_{\mathrm{X}}=2 \times 10^{35} \mathrm{ergs} \mathrm{s}^{-1}$ (Kallman et al. 2003), it is still interesting to explore the possible effects that X-rays may have on the secondary star's radial velocity curve. We do this using the X-ray binary model described by Phillips et al. (1999) and Shahbaz et al. (2000, 2003). We first phase-fold the radial velocities using the orbital ephemeris derived in $\S 3$, bin into 31 orbital phase bins, and fit the resulting curve with the model. We consider two extreme cases for the X-ray irradiation: case $a$, in which the X-rays produced near the compact object irradiate the whole inner face of the secondary star, and case $b$, in which the X-rays do not affect the secondary star. Note that invoking a flared accretion disk will have intermediate solutions. The model parameters are the compact object mass $M_{1}$, secondary star mass $M_{2}, \mathrm{X}$-ray luminosity $L_{\mathrm{X}}$, and the inclination $i$. To determine which elements on the star contribute to the absorption-line radial velocity, we use the factor $f_{\mathrm{x}}$, which is the fraction of the external radiation flux that exceeds the unperturbed flux. For a given $i$ and combination of $M_{1}$ and $M_{2}$, we fit the data and use the constraints imposed by $v \sin i$ to produce plots in the $M_{1}-M_{2}$ plane (Fig. 3).

Given the evidence for optical and X-ray partial eclipse, $i$ must lie in the range $70^{\circ}-90^{\circ}$. Although the lack of X-ray dips suggests $i>80^{\circ}$ (Frank et al. 1987), we prefer to adopt a more conservative range, since $i$ has not been measured accurately. Therefore, we consider cases $a$ and $b$ for $i=70^{\circ}$ and, more importantly, $i=90^{\circ}$, because it gives a firm lower limit to $M_{1}$. For case $a$ we fix $L_{\mathrm{X}}=2 \times 10^{35} \mathrm{ergs} \mathrm{s}^{-1}$ and find that the best fit $\left(\chi^{2}=122\right)$ is obtained when all the elements on the inner face contribute to the radial velocity, i.e., $f_{\mathrm{X}}>60 \%$, i.e., $\mathrm{X}$-ray heating is not preferred. Note, however, that the radial velocity curve will not be sinusoidal because there will still be some irradiated elements that contribute to the radial velocity. Even if we increase to $L_{\mathrm{X}}=10^{39} \mathrm{ergs} \mathrm{s}^{-1}$ as could be the case for an LMXB, the fits are significantly worse $\left(\chi^{2}=190\right)$, and the best fit still occurs when all the inner face contributes to the radial velocity. For case $b$ we set $L_{\mathrm{X}}=0$, implying no Xray heating, and find that it gives a fit with similar quality $\left(\chi^{2}=119\right)$. We conclude that the best fit to the radial velocity curve is the case in which the X-rays do not have an observable effect given the quality of the data. For our range in $i$, our fits for cases $a$ and $b$ give $2.0 M_{\odot}<M_{1}<4.3 M_{\odot}(1 \sigma)$, which means we can rule out a canonical $1.4 M_{\odot}$ neutron star at the 99\% level. $M_{1}$ is either a massive neutron star or a low-mass black hole; the maximum possible neutron star mass is $2.9 M_{\odot}$ (Kalogera \& Baym 1996).

Since the donor star fills its Roche lobe and is synchronized with the binary motion, we use $v \sin i$ and $K_{2}$ to determine the binary mass ratio $q=\left(M_{2} / M_{1}\right)=0.89 \pm 0.18$ (Horne et al. 1986). Given the measured masses, the temperature inferred from the spectral type and $P_{\text {orb }}$, we can determine the semimajor axis $56.1 R_{\odot}<a<72.4 R_{\odot}$, the radius $10.4 R_{\odot}<R_{2}<13.4 R_{\odot}$, and luminosity $52.5 L_{\odot}<L_{2}<87.1 L_{\odot}$ of the secondary star $(1 \sigma)$.

\section{DISCUSSION}

The position of the secondary star of $2 \mathrm{~S} 0921-630$ in an $\mathrm{H}-\mathrm{R}$ diagram corresponds to a normal star that has crossed the Hertzsprung gap and now lies on the Hayashi line. The evolution of the binary is dominated by the evolution of the evolved secondary. Since such a star no longer burns hydrogen in its core, the mass transfer is early massive case B. For a binary with a secondary star near the onset of such mass transfer, one requires $q<1$ throughout its history (King \& Ritter 1999). The case for a $1.4 M_{\odot}$ neutron star can be ruled out since it requires $M_{2}<1.4 M_{\odot}$, which is inconsistent with our results; the position of a secondary on the H-R diagram un- 
dergoing early massive case B mass transfer is close to that of a single star of the same mass (Kolb 1998). For $M_{1}=3 M_{\odot}$, $M_{2}<3.0 M_{\odot}$, which is consistent with our observations. It is interesting to note that the $q$ that we derive lies on the limit of stable mass transfer $q=\frac{5}{6}$ (King \& Ritter 1999). It is possible that $2 \mathrm{~S} 0921-630$ is undergoing unstable mass transfer similar to the supersoft sources and other X-ray binaries (Kahabka \& van den Heuvel 1997).

The distribution of measured neutron star masses provides fundamental constraints on the equation of state of nuclear matter and the observational identification of a black hole, which is based on the maximum possible mass allowed for a neutron star, 2.9 $M_{\odot}$ (Kalogera \& Baym 1996). There is some evidence that neutron stars with a mass in excess of $1.4 M_{\odot}$ do exist. LMXBs are expected to contain massive $\left(\sim 2 M_{\odot}\right)$ neutron stars, resulting from the accretion of a considerable amount of material over extended $\left(10^{8} \mathrm{yr}\right)$ periods of time (Zhang et al. 1985). However, while a neutron star mass of $1.78 \pm 0.23 M_{\odot}$ for the LMXB Cyg X-2 is reported (Orosz \& Kuulkers 1999), Titarchuk \& Shaposhnikov (2002) have recently proposed a mass of $1.44 \pm 0.06 M_{\odot}$ based on the spectral and temporal properties of the type I X-ray bursts. The analysis of optical data for the X-ray pulsar Vela X-1 suggests that the pulsar has a mass of $1.87_{-0.17}^{+0.23} M_{\odot}$ (Barziv et al. 2002). Probably the strongest case for a massive neutron star or lowmass black hole is in the eclipsing HMXB 4U 1700-37, which contains a $2.44 \pm 0.27 M_{\odot}$ compact object (Clark et al. 2002). Such masses already test soft nuclear equations of state (Miller et al. 1998b). We obtain $M_{1}=2.0-4.3 M_{\odot}$ in 2S 0921-630, which lies between the range of masses observed for neutron stars and black holes. Models for nuclear equations of state, which include the effects of three nucleon interactions and realistic models of nuclear forces, limit the maximum mass of neutron stars to less than $2.5 M_{\odot}$ (Akmal et al. 1998). However, it should be noted that unconventional forms of matter such as Q stars do allow the existence of extremely massive neutron stars (Miller et al. 1998a). Although our results do not allow us to distinguish between a massive neutron star and a low-mass black hole, the existence of a greater than $2.5 M_{\odot}$ neutron star would place strong constraints on high-density nuclear matter.

Theoretical predictions suggest that the death of a $60 M_{\odot}$ star in a close binary can produce anything from a low-mass $1.2 M_{\odot}$ neutron star to a $10 M_{\odot}$ black hole, depending on the wind mass-loss rate during the Wolf-Rayet phase (Fryer \& Kalogera 2001). The formation of a low-mass black hole with a high space velocity is possible via a two-stage process involving the formation of a neutron star (Brown et al. 1996). However, if there is symmetric mass ejection in the supernova, then a kick at the formation of a black hole is not required to explain the varied range in the observed space velocities of the black hole LMXBs (Nelemans et al. 1999).

The unusual mass for the compact object in 2S 0921-630, and the fact that the nature of the compact object is not clear, conjures up many formation scenarios. If it is a neutron star, then its present mass can be explained by either the accumulation of matter (1-2 $\left.M_{\odot}\right)$ by a canonical $1.4 M_{\odot}$ neutron star or the direct collapse of a massive star forming a massive neutron star. If the compact object in $2 \mathrm{~S} 0921-630$ is a black hole, then its low mass can be explained by the accretion-induced collapse of a massive $2.9 M_{\odot}$ neutron star or the formation of a low-mass black hole (Brown et al. 1996). Given the fact that we do not observe a peculiar systemic velocity comparable to other neutron star LMXBs, this suggests that we can rule out canonical neutron star formation scenarios in which a kick is produced during a Type II supernova. The most likely scenario for the formation of the compact object is the direct formation of a massive neutron star or a low-mass black hole.

T. S. and J. C. acknowledge support from the program Ramón y Cajal. R. I. H. is currently supported by NASA through Hubble Fellowship grant HF-01150.01-A awarded by the STSci, which is operated by AURA, Inc., for NASA under contract NAS 5-26555. D. S. acknowledges an SAO Clay Fellowship. The PAMELA and MOLLY routines of K. Horne and T. R. Marsh are gratefully acknowledged. This Letter uses observations made at the SAAO.

\section{REFERENCES}

Akmal, A., Pandharipande, V. R., \& Ravenhall, D. G. 1998, Phys. Rev. C, 58, 1804

Barziv, O., Kaper, L., van Kerkwijk, M. H., Telting, J. H., \& van Paradijs, J. 2001, A\&A, 377, 925

Branduardi-Raymont, G., Corbet, R. H. D., Mason, K. O., Parmar, A. N., Murdin, P. G., \& White, N. E. 1983, MNRAS, 205, 403

Brown, G. E., Weingartner, J. C., \& Wijers, R. A. M. J. 1996, ApJ, 463, 297

Casares, J., Charles, P. A., \& Kuulkers, E. 1998, ApJ, 493, L39

Casares, J., Dubus, G., Shahbaz, T., Zurita, C., \& Charles, P. A. 2002, MNRAS, 329,29

Casares, J., Steeghs, D., Hynes, R. I., Charles, P. A., \& O’Brien, K. 2003, ApJ, 590, 1041

Charles, P. A., \& Coe, M. 2004, in Compact Stellar X-Ray Sources, ed. W. H. G. Lewin \& M. van der Klis (Cambridge: Cambridge Univ. Press), in press (astro-ph/0308020)

Chevalier, C., \& Ilovaisky, S. A. 1982, A\&A, 112, 68

Clark, J. S., Goodwin, S. P., Crowther, P. A., Kaper, L., Fairbairn, M., Langer, N., \& Brocksopp, C. 2002, A\&A, 392, 909

Cowley, A. P., Crampton, D., \& Hutchings, J. B. 1982, ApJ, 256, 605

Dehnen, W., \& Binney, J. 1998, MNRAS, 294, 429

Frank, J., King, A. R., \& Lasota, J.-P. 1987, A\&A, 178, 137

Fryer, C. L., \& Kalogera, V. 2001, ApJ, 554, 548

Horne, K., Wade, R. A., \& Szkody, P. 1986, MNRAS, 219, 791

Kahabka, P., \& van den Heuvel, E. P. J. 1997, ARA\&A, 35, 69

Kallman, T. R., Angelini, L., Boroson, B., \& Cottam, J. 2003, ApJ, 583, 861
Kalogera, V., \& Baym, G. 1996, ApJ, 470, L61

King, A. R., \& Ritter, H. 1999, MNRAS, 309, 253

Kolb, U. 1998, MNRAS, 297, 419

Li, F. K., Clark, G. W., Jernigan, J. G., Laustsen, S., Zuiderwijk, E. J., \& van Paradijs, J. A. 1978, Nature, 276, 799

Mason, K. O., Branduardi-Raymont, G., Codova, F. A., \& Corbet, R. H. D. 1987, MNRAS, 226, 423

Miller, J. C., Shahbaz, T., \& Nolan, L. A. 1998a, MNRAS, 294, L25

Miller, M. C., Lamb, F. K., \& Psaltis, D. 1998b, ApJ, 508, 791

Nelemans, G., Tauris, T. M., \& van den Heuvel, E. P. J. 1999, A\&A, 352, L87

Orosz, J. A., \& Kuulkers, E. 1999, MNRAS, 305, 132

Phillips, S. N., Shahbaz, T., \& Podsiadlowski, P. 1999, MNRAS, 304, 839

Shahbaz, T., Groot, P., Phillips, S. N., Casares, J., Charles, P. A., \& van Paradijs, J. 2000, MNRAS, 314, 747

Shahbaz, T., Kuulkers, E., Charles, P. A., van der Hooft, F., Casares, J., \& van Paradijs, J. 1999, A\&A, 344, 101 (S99)

Shahbaz, T., Zurita, C., Casares, J., Dubus, G., Charles, P. A., Wagner, R. M., \& Ryan, E. 2003, ApJ, 585, 443

Titarchuk, L., \& Shaposhnikov, N. 2002, ApJ, 570, L25

Torres, M. A. P., Casares, J., Martínez-Pais, I. G., \& Charles, P. A. 2002, MNRAS, 334, 233

van Kerkwijk, M. H. 2001, in Black Holes in Binaries and Galactic Nuclei, ed.

L. Kaper, E. P. J. van den Heuvel, \& P. A. Woudt (Berlin: Springer), 39

Zhang, W., Strohmayer, T. E., \& Swank, J. H. 1997, ApJ, 482, L167 